\title{
Third-generation cholecystectomy by natural orifices: transgastric and transvesical combined approach (with video) $\square$
}

Carla Rolanda, MD, Estêvão Lima, MD, José M. Pêgo, MD, Tiago Henriques-Coelho, MD, David Silva, MD, Ivone Moreira, Guilherme Macedo, MD, PhD, José L. Carvalho, MD, Jorge Correia-Pinto, MD, PhD

Braga, Portugal

Background: An isolated transgastric port has some limitations in performing transluminal endoscopic cholecystectomy. However, transvesical access to the peritoneal cavity has recently been reported to be feasible and safe.

Objective: To assess the feasibility and the technical benefits of transgastric and transvesical combined approach to overcome the limitations of isolated transgastric ports.

Design: We created a transgastric and transvesical combined approach to perform cholecystectomy in 7 consecutive anesthetized female pigs. The transgastric access was achieved after perforation and dilation of the gastric wall with a needle knife and with a balloon, respectively. Under cystoscopic control, an ureteral catheter, a guidewire, and a dilator of the ureteral sheath were used to place a transvesical 5-mm overtube into the peritoneal cavity. By using a gastroscope positioned transgastrically and a ureteroscope positioned transvesically, we carried out cholecystectomy in all animals.

Results: Establishment of transvesical and transgastric accesses took place without complications. Under a carbon dioxide pneumoperitoneum controlled by the transvesical port, gallbladder identification, cystic duct, and artery exposure were easily achieved in all cases. Transvesical gallbladder grasping and manipulation proved to be particularly valuable to enhance gastroscope-guided dissection. With the exclusion of 2 cases where mild liver-surface hemorrhage and bile leak secondary to the sliding of cystic clips occurred, all remaining cholecystectomies were carried out without incidents.

Limitations: Once closure of the gastric hole proved to be unreliable when using endoclips, the animals were euthanized; necropsy was performed immediately after the surgical procedure.

Conclusions: A transgastric and transvesical combined approach is feasible, and it was particularly useful to perform a cholecystectomy through exclusive natural orifices. (Gastrointest Endosc 2007;65:111-7.)

Since the first reports in the late 1980s, laparoscopy has progressively become the criterion standard for cholecystectomy, one of the most widely performed abdominal interventions in developed countries. In fact, minimally invasive surgery is now associated with many proven advantages over traditional open procedures, such as minimal scarring, reduced pain, and faster patient recovery. ${ }^{1}$

In parallel with the progression of minimally invasive surgery, improvements in endoluminal endoscopy have made it an indispensable and multifaceted instrument for diagnosis and, definitively, for therapy. ${ }^{2}$ Recently, Reddy and Rao (N. Reddy, V. G. Rao, oral communications,

Copyright $\odot 2007$ by the American Society for Gastrointestinal Endoscopy 0016-5107/\$32.00

doi:10.1016/j.gie.2006.07.050
May 2005; N. Reddy, oral communication, May 2004), in human beings, and Kalloo et $\mathrm{al}^{3}{ }^{3}$ in a porcine model, described a new port to the peritoneal cavity through a transgastric approach. Subsequently, various investigators described more complex intra-abdominal procedures in porcine model, ${ }^{4-12}$ opening a new era in the surgical field in what seems to be the third-generation surgery: natural orifice transluminal endoscopic surgery (NOTES). ${ }^{13}$

Park et $\mathrm{al}^{5}$ conducted the first pilot study in pigs by applying NOTES to perform transgastric cholecystectomy. By using 2 endoscopes or a single endoscope conjugated with a transabdominal trocar, cholecystectomy was feasible, but important limitations were identified. These were related to difficulty in controlling the pneumoperitoneum and in obtaining a stable platform for anatomy exposure, organ retraction, secure grasping, and adequate 
triangulation of instruments. Swanström et $\mathrm{al}^{12}$ attempted to overcome these limitations by using ShapeLock technology (USGI Medical, San Clement, Calif) as a new overtube for transgastric surgery. However, even with this equipment, an isolated transgastric approach for gallbladder manipulation remained a challenge, with only a $33.3 \%$ success rate. ${ }^{12}$

These studies were able to demonstrate that cholecystectomy may one day be performed without skin incisions. The development of other natural orifice accesses, however, may play an important role in overcoming some of the limitations identified for those who performed abdominal surgery through isolated transgastric surgery. ${ }^{13}$ By applying these concepts, Lima et $\mathrm{al}^{14}$ demonstrated that transvesical endoscopic peritoneoscopy was technically feasible and could be safely performed in a porcine model. By using a transvesical port, it was possible to introduce 5-mm rigid instruments, such as graspers, scissors, and telescopes. We hypothesized that a transvesical port could be useful to perform abdominal procedures in combination with a transgastric pathway.

The purpose of this experimental study was to assess the feasibility and the technical benefits of a combined transgastric and transvesical approach for cholecystectomy in a porcine model.

\section{MATERIALS AND METHODS}

This was a nonsurvival study approved by the ethical review boards of Minho University (Braga, Portugal). For this study, we included 15 small $(15-25 \mathrm{~kg})$ female pigs (Sus scrofus domesticus) so that the current ureteroscope length could easily achieve the upper-abdominal organs. After a significant surgical and anesthetic learning curve, the results of which are not included in this report (9 animals), we performed the cholecystectomy exclusively through natural orifices (transgastric and transvesical combined approach) in 7 consecutive animals. After the surgical procedures, the animals were euthanized, and necropsies were performed.

\section{Pig preparation}

The animals were fed liquids for 3 days and then were restrained from food ( 24 hours) and water ( 6 hours) before the surgical intervention. All procedures were performed with the pigs under general anesthesia, with 5.0-mm endotracheal intubation and mechanical ventilation. Preanesthesia medication consisted of an intramuscular injection of $32 \mathrm{mg} / \mathrm{mL}$ azaperone (Stressnil; Esteve Farma, Barcelona, Spain) reconstituted with $1 \mathrm{mg} / \mathrm{mL}$ midazolam (Dormicum; Roche, Amadora, Portugal) at a dose of 0.15 to $0.2 \mathrm{~mL} / \mathrm{kg}$.

Venous access was obtained through an intravenous line placed in the marginal ear vein. Anesthesia was induced with $3 \mu \mathrm{g} / \mathrm{kg}$ fentanyl (Fentanest; Janssen-Cilag,

\section{Capsule Summary}

\section{What is already known on this topic}

- Transgastric cholecystectomy is feasible in a porcine model, but it has limitations that jeopardize its clinical application, including difficulty in controlling the pneumoperitoneum and in securing a stable platform for anatomy exposure, organ retraction, secure grasping, and adequate instrument triangulation.

\section{What this study adds to our knowledge}

- In 7 pigs, gallbladder identification and cystic duct and artery exposure were achieved by using transvesical and transgastric access; transvesical gallbladder grasping and manipulation was particularly useful in gastroscopeguided dissection.

Queluz, Portugal), $10 \mathrm{mg} / \mathrm{kg}$ thiopental sodium (Pentothal; Abbott, Alfragide, Portugal), and $1 \mathrm{mg} / \mathrm{kg}$ vecuronium (Norcuron; Organon, Oss, The Netherlands). Anesthesia was maintained with $1.5 \%$ to $2 \%$ sevoflurane (Sevorane; Abbott) and a perfusion of $1 \mathrm{mg} / \mathrm{kg}$ per hour of vecuronium. All animals received an intramuscular injection of $1 \mathrm{~g}$ ceftriaxone (Rocephin; Roche) before beginning the surgical procedures.

\section{Surgical technique}

Transvesical access. Creation of a transvesical access is illustrated in Video 1 (available online at http://www. giejournal.org). An ureteroscope (A2942A; Olympus, Tokyo, Japan) was introduced through the urogenital sinus and the urethra into the bladder with hydrodistension. Before any further procedure, the bladder was emptied of urine and refilled with saline solution. The vesicotomy site was carefully selected on the ventral bladder wall, posterior to the bladder dome. A mucosal incision was made with a scissors (A2576; Olympus) introduced by the working channel of the ureteroscope. Subsequently, a $5 \mathrm{~F}$ openend ureteral catheter (Selectip, 62450200; Angiomed, Bard, Murray Hill, NJ) was pushed forward through the incision into the peritoneal cavity. A 0.035 -inch flexible-tip guidewire (RF*GA35153M, Terumo Corp, Somerset, NJ) was then inserted into the peritoneal cavity through the lumen of the ureteral catheter. Guided by the flexible-tip guidewire, the vesical hole was enlarged with a dilator of an ureteroscope sheath (250-105; Microvasive Endoscopy, Boston Scientific Corp, Natick, Mass), which was enveloped with an overtube designed by us $(25-\mathrm{cm}$ length, $5.5-\mathrm{mm}$ internal diameter, and 1-mm wall thickness). A rigid ureteroscope was introduced into the peritoneal cavity within the overtube and allowed the creation of a pressure-controlled carbon dioxide $\left(\mathrm{CO}_{2}\right)$ pneumoperitoneum up to $12 \mathrm{mmHg}$. Through the overtube, we could insert into the peritoneal cavity either a video telescope with 
5-mm diameter, chip-on-the-tip, and 0-degree view direction, normally used as a laparoscope (EndoEye 50021A; Olympus) or a rigid ureteroscope that includes a working channel. The peritoneal cavity was thoroughly examined, with particular emphasis on the stomach, the liver, and the gallbladder.

Transgastric access. An adult forward-viewing, double-channel endoscope (GIF-2T160; Olympus) was advanced into the esophagus and the stomach. The stomach was lavaged with instilled water and was aspirated through the gastroscope until free of food particles. Subsequently, it was decompressed and a cefazolin solution (1 g in $200 \mathrm{~mL}$ saline solution) was instilled. The antibiotic solution was left in the stomach for 10 minutes before also being aspirated. For the gastrotomy-site selection and to avoid damage of the gastric vessels or surrounding organs, the gastroscope operator was working with both internal (provided by the gastroscope observing the internal stomach indentation produced by external abdominal palpation) and external (provided by the EndoEye or ureteroscope) gastric-wall images. The gastrotomy site was carefully chosen on the anterior wall (bodyantrum transition). A gastric-wall incision was made by pushing forward a needle knife, followed by its sheath (KD-11Q-1; Olympus) with cautery (PSD 20; Olympus) under a $12 \mathrm{mmHg} \mathrm{CO}_{2}$ pneumoperitoneum (induced through the transvesical port). The needle-knife sheath was then used for positioning a guidewire (5156-01; Microvasive Endoscopy). The puncture dilation was performed with an 18-mm through-the-scope balloon (5837; Microvasive Endoscopy) over the guidewire. On balloon semideflection, the gastroscope was pushed forward and passed into the peritoneal cavity. All these procedures were monitored by the scope positioned through the transvesical port.

\section{Cholecystectomy surgical procedure}

After establishment of the 2 diametrically opposed ports (transgastric and transvesical) and $\mathrm{CO}_{2}$-pneumoperitoneum creation, the ureteroscope easily identified the gallbladder. A forceps was then passed through the ureteroscope working channel, and the gallbladder fundus was grasped and upward retracted. This maneuver nicely exposed the gallbladder infundibulum and the cystic duct. Subsequently, the gastroscope was moved on retroflexion toward the gallbladder. The exposure achieved by the transvesical gallbladder grasping allowed the gastroscope operator to quickly identify the cystic duct. This allowed us to start careful dissection, handling either a grasping forceps (FG-6L-1, FG-47L-1; Olympus) or a $2.8-\mathrm{mm}$ ball coagulation electrode (CD-1U; Olympus) passed through the working channels of the gastroscope. For this purpose, we also had available a pre-cut needle knife (KD-11Q-1; Olympus) and a scissors (FS-5L-1; Olympus). Both sides of cystic duct were completely dissected by alternating the working channels of the grasping forceps and the coagulation electrode. When the cystic duct and artery were dissected and free, 3 clips (HX200L-135; Olympus) were applied: 2 on the gallbladder extremity and the other proximally. Sectioning of pedicle was then carried out with a needle knife. The grasping provided by the ureteroscope allowed us to retract the gallbladder body in the major axis (up and down; right and left), looking for the most appropriate anatomical exposure for dissection. Small position adjustments were also possible when using the forceps introduced by the gastroscope. Blunt dissection was taken progressively by electrocautery of the gallbladder bed. When cholecystectomy was completed, the gastroscopic forceps held the cystic-duct extremity distally to clips, and the gallbladder was removed via the esophagus and the mouth.

\section{RESULTS}

All procedures involved in the creation of the vesical hole (cystoscopy, bladder mucosal incision, vesicotomy, transvesical overtube passage) were performed without complications. The ureteroscope was easily introduced into the peritoneal cavity, and insufflation of $\mathrm{CO}_{2}$ was performed without incident. By using the transvesical port, we could obtain a perfect view of the upper-abdominal organs (liver, gallbladder, stomach, spleen, and diaphragm). This was particularly useful in helping the gastroscope operator to safely perform the gastrotomy.

Under a $\mathrm{CO}_{2}$ pneumoperitoneum and with the view provided by the transvesical port, we created the gastrotomy in a rapid, safe, and easy way. In fact, with our approach, neither gastric vessels nor surrounding organs were damaged. In addition, the ureteroscope was extremely useful many times in assisting the passage of the gastroscope through the gastrotomy.

Gallbladder identification was easily achieved in all experiments, first, by the ureteroscope. After gallbladder fundus grasping with transvesical instruments, the gastroscope easily identified the cystic duct in all cases (Fig. 1). Under transvesical gallbladder retraction, gastroscopic dissection (Fig. 2), isolation, clipping, and sectioning (Fig. 3) of the cystic duct and artery was feasible and, therefore, was performed in a precise way in all cases. For these purposes, we successfully used a grasping forceps and a coagulation electrode inserted through the gastroscope working channels. A pre-cut needle knife and scissors were rarely applied in these procedures. After sectioning of the clipped cystic duct and artery, we began a dissection of the gallbladder from its bed by using the coagulation electrode (Fig. 4). This proved to be the most fastidious and, simultaneously, the most challenging part of the surgery, because the gallbladder body is commonly hidden in the liver parenchyma in pigs. Nevertheless, the transvesical grasping allowed significant manipulation of the gallbladder (Fig. 5), which was particularly useful in 

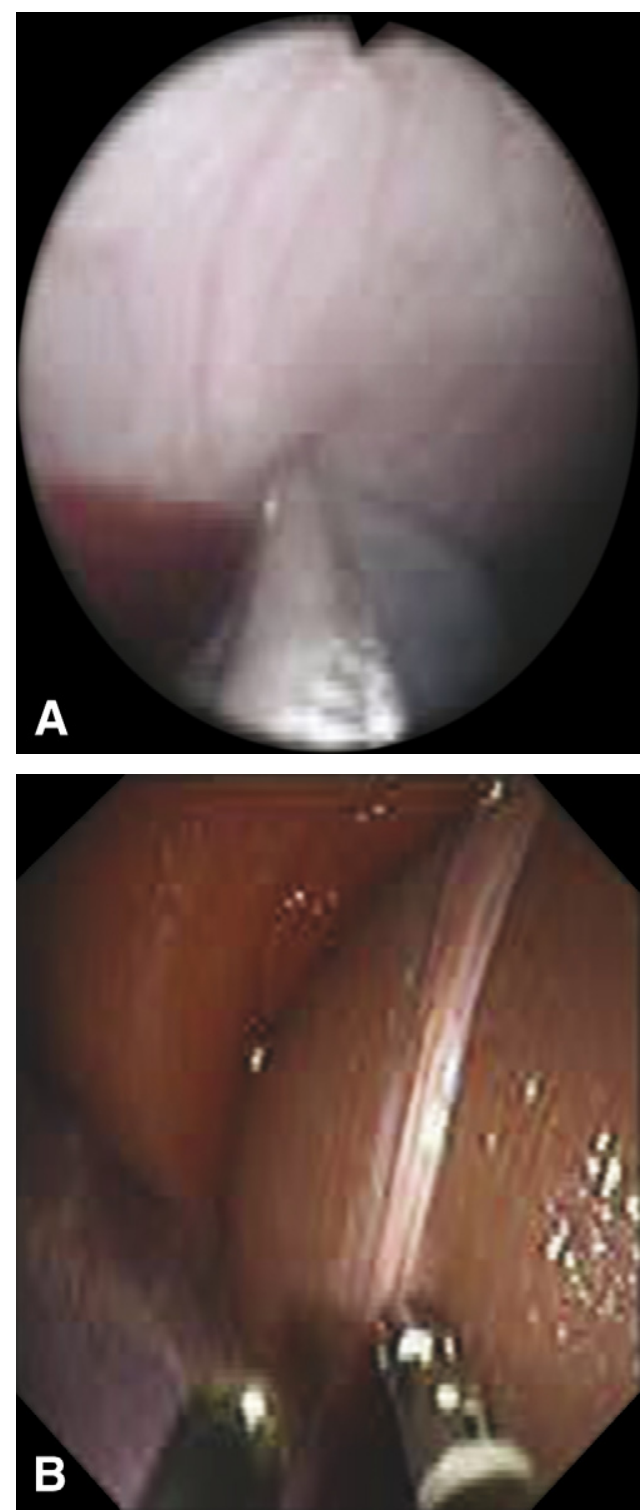

Figure 1. Exposure of cystic duct and artery. A, Ureteroscope image: the ureteroscopic forceps holds the gallbladder fundus. B, Gastroscope image: transvesical gallbladder retraction exposes the cystic duct and artery for the gastroscope.

selecting the best position for the gastroscope-guided dissection. Liver-surface bleeding occurred in only one case, where an oozing hemorrhage, which did not obscure endoscopic visualization, became significant after relief of the gallbladder upward retraction. We also reported the sliding of a cystic clip and a secondary bile leak as a complication in another case, but, in this circumstance, bubbles from bile spillage seriously disturbed endoscopic view.

Coordination of gallbladder transvesical-transgastric manipulation proved feasible, with rapid progress during the protocol. Similarly, gallbladder withdrawal and retrograde mouth exteriorization were easily achieved with a grasper through the gastroscope. The median time for

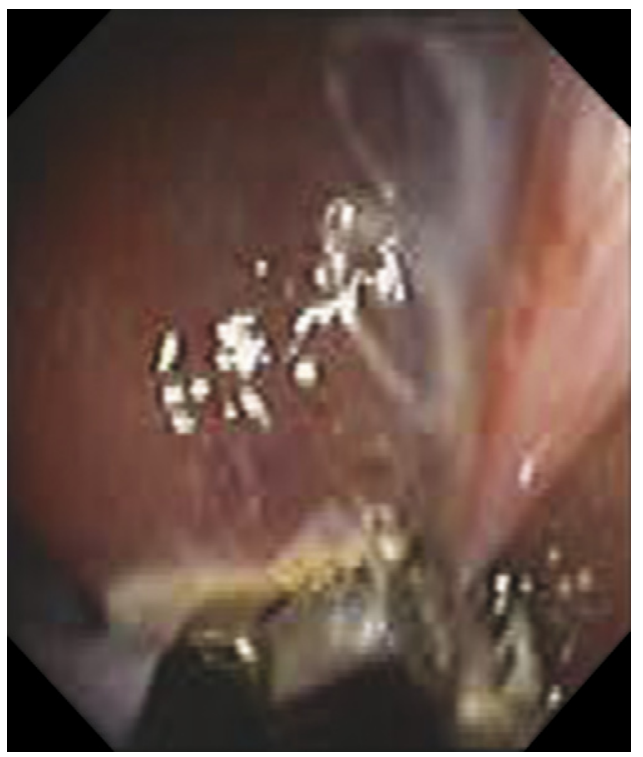

Figure 2. Dissection of the cystic duct and artery. After upward gallbladder retraction (by transvesical port), small position adjustments were possible by using the forceps introduced by the gastroscope.

the overall procedure, including establishment of transvesical and transgastric ports, was 2 hours.

During preliminary experiments performed before starting this study, the closure of a gastric perforation with endoscopic clips were shown, to us, to be unreliable. For this reason, we did not carry out any attempts of gastric closure, and all the animals were euthanized at the end of the cholecystectomy procedure. Necropsies did not reveal any damage of the intraperitoneal organs related to the transgastric or transvesical access and manipulation, except mild hemoperitoneum and bile peritoneal spillage in the above referred cases. The gastric holes measured, on average, $15 \mathrm{~mm}$.

\section{DISCUSSION}

Almost 20 years after the first human laparoscopic cholecystectomy, a procedure that was initially viewed with skepticism, many advances have occurred in surgery. ${ }^{1}$ Similarly, with the recent unexpected success of a transgastric approach in porcine model, ${ }^{3-12}$ an exciting new frontier in minimally invasive surgery was born and is reenergizing the surgical world: NOTES. Whereas, laparoscopic advantages over the open cholecystectomy are well recognized, many potential benefits of cholecystectomy by NOTES over laparoscopy are predicted: (i) avoidance of abdominal scars; (ii) less painful procedure; (iii) possible avoidance of general anesthesia; (iv) probably a preferable approach for morbid obese patients or with scars, burns, and infections in the abdominal wall; and (v) avoidance of postoperative hernias.

When trying to reproduce previous descriptions of transgastric cholecystectomy, ${ }^{5}$ we experienced several 


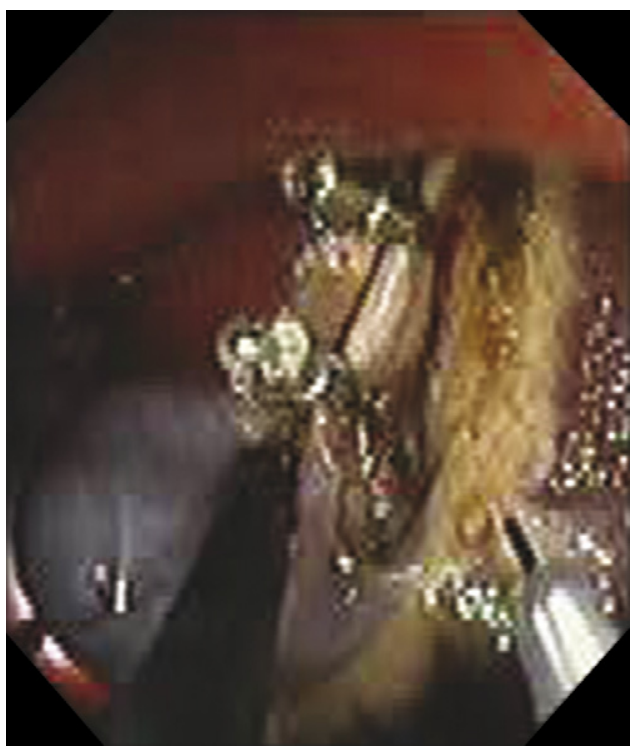

Figure 3. Cystic duct and artery clipping. After cystic duct and artery dissection, endoscopic clips were easy to apply allowing sectioning by a needle knife.

problems, as highlighted by others. ${ }^{5,12}$ This gave us the rationale to predict that an additional transvisceral port diametrically opposed to the stomach would be helpful in performing complex upper-abdominal endoscopic procedures. In this sequence, we recently proved that a transvesical access through a 5-mm port was technically feasible and safe in a survival porcine model. ${ }^{14}$ In the current study, we tested the feasibility and the technical utility of using 2 opposed ports; rigid instruments were used in one to perform cholecystectomy.

Gallbladder identification and cystic pedicle exposure have been reported as a challenge when using an exclusive transgastric port. ${ }^{5,7,12}$ Interestingly, with our approach, we could easily identify the gallbladder and expose the cystic duct and artery in all cases. In fact, the frontal view provided by the transvesical access was a determinant for these achievements, because it allowed us to rapidly identify gallbladder fundus, usually without needing special transgastric intervention. Furthermore, the gallbladder upward retraction accomplished by the transvesical operator easily exposed the cystic duct and artery for the gastroscope.

Efficient gallbladder retraction was clearly enhanced by using rigid instruments through the transvesical port. The possibility to introduce rigid instruments with direct handling reinforces the role of a transvesical port in NOTES procedures. One of the major limitations of transgastric surgery was the inexistence of a stable platform that permits organ retraction and triangulation for gallbladder dissection and manipulation. ${ }^{13,15}$ This limitation was attributed to the flexibility of current gastroscopes that avoid robust grasping and retraction. In fact, we also had an opportunity to verify that the gastroscope in the retroflexion position and unsupported in the pneumoper-

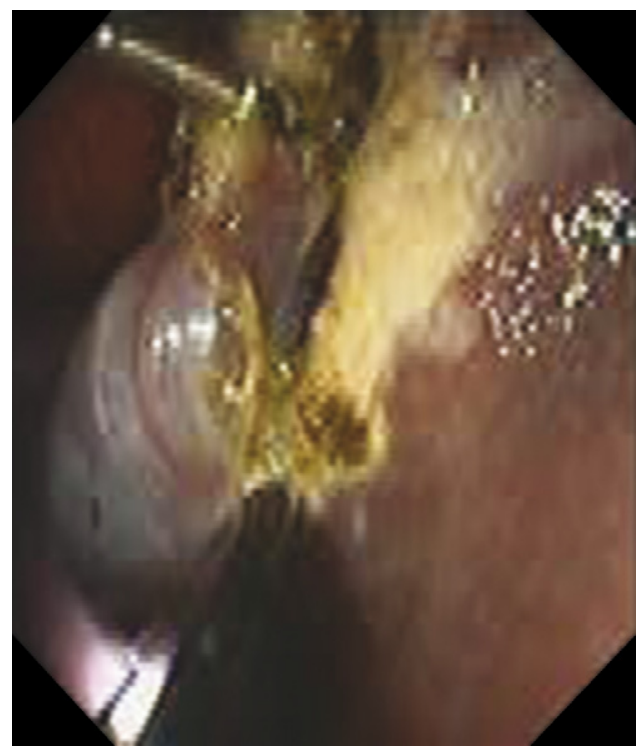

Figure 4. Beginning of gallbladder-bed dissection. By using electrocautery, gallbladder bed dissection was carefully undertaken step-by-step.

itoneum was unreliable for simultaneously exerting organ retraction and dissection. To overcome this limitation, Park et $\mathrm{al}^{5}$ used either an additional gastroscope or a transabdominal trocar, whereas Swanström et al ${ }^{12}$ used flexible multilumen guides that can be locked in position. Even in these circumstances, cholecystectomy was accomplished in only $33 \%$ of the attempts. Our transvesical port allowed the passage of a rigid forward-viewing instrument with a forceps that permitted efficient grasping and retraction. In addition, it made it possible to mobilize the gallbladder in various axes, exposing different areas for gastroscopeguided dissection. This partially overcame the absence of triangulation experienced when using only a transgastric port. Although we still needed to work in a retroflexion position, the good exposure achieved by coordinated movements of transvesical devices allowed us to straightforwardly use instruments through both the gastroscope working channels, minimizing the need of gastroscopedependent grasping.

Another advantage of using the transvesical port was the possibility to work under a pressure controlled $\mathrm{CO}_{2}$ pneumoperitoneum. This overcomes some common consequences of pneumoperitoneum created by the gastroscope, such as the detrimental action of high ( $>15 \mathrm{~mm} \mathrm{Hg}$ ) intraperitoneal pressures, the augmented combustion risk, and the slower air reabsorption rate. ${ }^{13}$ In fact, insufflation provided by current flexible endoscopes is neither pressure controlled nor uses $\mathrm{CO}_{2}$

Insufflation of the pneumoperitoneum before gastrotomy creation proved useful in preventing undesired damage of gastric vessels and adjacent organs. In addition, with the transvesical image, we could monitor all procedures involved in the gastrotomy creation. Unintended and unrecognized laceration of adjacent organs is a major 

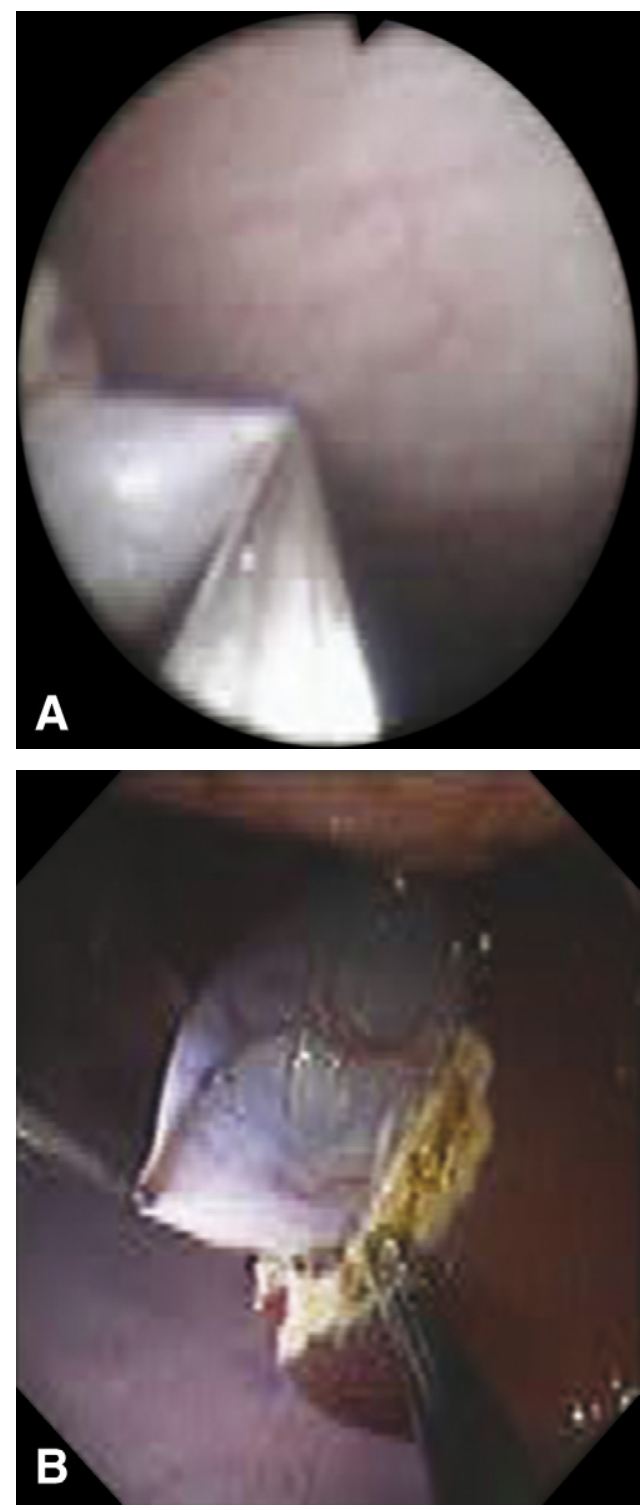

Figure 5. Coordinated transgastric-transvesical approach. A, Ureteroscope image, and $\mathbf{B}$, gastroscope image, illustrating how transgastric and transvesical combined approach can easily mobilize the gallbladder.

concern reported by investigators dealing with transgastric approach. ${ }^{16}$ In fact, there are descriptions of liver and anterior abdominal-wall trauma during gastrotomy. ${ }^{10}$ The $\mathrm{CO}_{2}$ pneumoperitoneum and the open front view provided by the transvesical port allowed the displacement of the abdominal wall from the stomach and provided a good in-time control of the gastric exit of the gastroscope, making the procedure more rapid and safe. The risk of adjacent structure damage was not a problem during the creation of transvesical port, because it is created with atraumatic instruments and without electrocautery. In addition, because bowel loops in contact with the bladder wall (small intestine and sigmoid colon) are free in the abdomen, they run ahead of the ureteral catheter atraumatic tip.
Although this study reinforces that NOTES cholecystectomy is feasible, some important technical pitfalls remain unsolved. Gastric closure is likely the most important issue limiting widespread translation of NOTES for human beings. Although, we attempted to reproduce the technique of endoclip gastric closure executed by several investigators, ${ }^{3,5,7,10,11}$ we realized that endoclip application (currently available) was fastidious and cumbersome, with unreliable results and poor safety. This was the reason that we did not go forward with survival studies. We do not exclude the hypothesis that our technique for gastric perforation (balloon dilation) disturbed the conditions for clip application. In fact, we recognize that if dilation is efficient in maintaining muscle integrity for contraction, the cutting electrocautery would define more regular borders for tissue approximation. Even knowing gastric injuries heal quickly and that survival studies have been done, without any maneuver for gastric closure, with surprising good results, ${ }^{4}$ we believe that this is a major drawback that might risk the technique accreditation if not correctly surmounted. With regard to a transvesical port, we previously demonstrated in a survival study that a 5-mm transvesical hole closes spontaneously without complications in a porcine model. ${ }^{14}$ Bladder decompression by a vesical catheter and the healthiness of the bladder wall explain why vesicotomy suturing may not be necessary.

The risk of infection should not be neglected. Although neither bladder nor stomach are physiologically infected, the routes followed by the scopes may contaminate them. The development of appropriate overtubes, as already used by others ${ }^{4,11}$ for transgastric surgery, might significantly minimize this risk.

During our experiments with a transvesical port, we detected that the current instruments are too short to reach the upper abdomen. In our study, we dealt with this problem by using smaller animals, but we feel that biomedical engineering will need to develop longer instruments if we want to use the transvesical port in adult human beings.

In conclusion, this study demonstrated the usefulness of combining 2 natural orifices (transgastric and transvesical) ports in moderately complex abdominal surgical procedures, eg, cholecystectomy. The addition of the transvesical port overcame most of the limitations previously reported for those who attempted to perform cholecystectomy exclusively through a transgastric approach. This study reinforces the feasibility of exclusive natural orifices transluminal endoscopic cholecystectomy.

\section{ACKNOWLEDGMENTS}

We thank Mr Paulo Pereira and Mr José Bragança (Ethicon EndoSurgery, Portugal) for their assistance in this project. 


\section{DISCLOSURE}

The authors have no conflict of interest to disclosure.

\section{REFERENCES}

1. Harrell AG, Heniford T. Minimally invasive abdominal surgery: lux et veritas past, present, and future. Am J Surg 2005;190:239-43.

2. Liu R, Chand B, Ponsky J. The future of surgical endoscopy. Endoscopy 2005;37:38-41.

3. Kalloo AN, Singh VK, Jagannath SB, et al. Flexible transgastric peritoneoscopy: a novel approach to diagnostic and therapeutic interventions in the peritoneal cavity. Gastrointest Endosc 2004;60: 114-7.

4. Jagannath SB, Kantsevoy SV, Vaughn CA, et al. Per-oral transgastric ligation of fallopian tubes with long-term survival in a porcine model. Gastrointest Endosc 2005;61:449-53.

5. Park PO, Bergstrom M, Ikeda K, et al. Experimental studies of transgastric gallbladder surgery: cholecystectomy and cholecystogastric anastomosis (videos). Gastrointest Endosc 2005;61:601-6.

6. Kantsevoy SV, Jagannath SB, Niiyama H, et al. Endoscopic gastrojejunostomy with survival in a porcine model. Gastrointest Endosc 2005;62:287-92.

7. Wagh MS, Merrifield BF, Thompson CC. Endoscopic transgastric abdominal exploration and organ resection: initial experience in a porcine model. Clin Gastroenterol Hepatol 2005;3:892-6.

8. Kantsevoy SV, Hu B, Jagannath SB, et al. Transgastric endoscopic splenectomy. Surg Endosc 2006;20:522-5.

9. Bergstrom $M$, Ikeda K, Swain $P$, et al. Transgastric anastomosis by using flexible endoscopy in a porcine model. Gastrointest Endosc 2006;63:307-12.

10. Wagh MS, Merrifield BF, Thompson CC. Survival studies after endoscopic transgastric oophorectomy and tubectomy in a porcine model. Gastrointest Endosc 2006;63:473-8.
11. Merrifield BF, Wagh MS, Thompson CC. Peroral transgastric organ resection in the abdomen: feasibility study in pigs. Gastrointest Endosc 2006;63:693-7.

12. Swanström LL, Kozarek R, Pasricha PF, et al. Development of a new access device for transgastric surgery. J Gastrointest Surg 2005;8: 1129-37.

13. American Society for Gastrointestinal Endoscopy, SAGES. ASGE/SAGES Working Group on Natural Orifice Translumenal Endoscopic Surgery white paper, October 2005. Gastrointest Endosc 2006;63:199-203.

14. Lima E, Rolanda C, Pêgo JM, et al. Transvesical endoscopic peritoneoscopy: a novel $5 \mathrm{~mm}$ port for intra-abdominal scarless surgery. J Uro 2006;176:802-5.

15. Lamadé W, Hchberger J. Transgastric surgery: avoiding pitfalls in the development of a new technique [editorial]. Gastrointest Endosc 2006;63:698-700.

Received May 30, 2006. Accepted July 31, 2006

Current affiliations: Life and Health Sciences Research Institute, School of Health Sciences, University of Minho, Braga (C.R., E.L., J.M.P., D.S., G.M., J.C-P.); Departments of Gastroenterology and Anesthesiology, S. Marcos Hospital, Braga (C.R., J.M.P., G.M.); Department of Urology, St. António General Hospital, Porto (E.L.); Department of Oncology, Sra Oliveira Hospital, Guimarães (I.M.); Department of Pediatric Surgery, S. Joao Hospital, Porto (T.H-C., J.L.C., J.C-P.) Portugal.

This project was funded by grants: Bolsa de Investigação da Sociedade Portuguesa de Endoscopia Digestiva 2006, Bolsa de Investigação Básica JABA 2005 da Associação Portuguesa de Urologia, and Project POCI/SAUOBS/56428/2004 from FCT-Portugal.

Reprint requests: Jorge Correia-Pinto, MD, Instituto de Ciências da Vida e Saúde, Escola de Ciências da Saúde, Universidade do Minho, Campus de Gualtar, 4709-057 Braga, Portugal. 